\title{
Optimizing the Management of Cancer Patients Treated With Systemic Therapies During the COVID-19 Pandemic: The New Role of PCR and CT Scan
}

\author{
Alessandro A. Viansone ${ }^{1,2 *}$, Samy Ammari ${ }^{3}$, Laurent Dercle ${ }^{4}$ and Monica Arnedos ${ }^{1}$ \\ ${ }^{1}$ Breast Unit, Department of Medicine, Gustave Roussy Cancer Campus, Villejuif, France, ${ }^{2}$ Breast Unit-Oncology Unit, \\ Department of Medicine, University Hospital of Parma, Parma, Italy, ${ }^{3}$ Radiology Department, Gustave Roussy Cancer \\ Campus, Villejuif, France, ${ }^{4}$ Radiology Department, Columbia University Medical Center New York Presbyterian Hospital, \\ New York, NY, United States
}

OPEN ACCESS

Edited by: Samata Kakkad, Merck, United States

Reviewed by: Jacobo Rogado, Hospital Universitario Infanta Leonor, Spain Monika Engelhardt, University of Freiburg, Germany

*Correspondence: Alessandro A. Viansone alessandro.viansone@ gustaveroussy.fr

Specialty section: This article was submitted to Cancer Imaging and Image-directed Interventions, a section of the journal Frontiers in Oncology

Received: 09 May 2020 Accepted: 12 March 2021 Published: 28 May 2021

Citation:

Viansone AA, Ammari S, Dercle L and Arnedos $M$ (2021) Optimizing the Management of Cancer Patients Treated With Systemic Therapies During the COVID-19 Pandemic: The New Role of PCR and CT Scan.

Front. Oncol. 11:560585. doi: 10.3389/fonc.2021.560585
In late 2019 and early 2020, the world witnessed the outbreak of the SARS-CoV-2 (also referred as COVID-19) in Wuhan, China. Its rapid expansion worldwide and its contagiousness rate have forced the activation of several measures to contain the pandemic, mostly through confinement and identification of infected patients and potential contacts by testing.

Keywords: COVID-19, PCR, CT-scan, oncology, cancer

\section{INTRODUCTION}

It is well established that patients with cancer are more susceptible to infections since they tend to be older, have multiple comorbidities, and because of the immunosuppressive state caused by anticancer treatments (1), so they could be potentially at particular risk from COVID-19 infection (2).

In a Chinese analysis (3) Professor He and colleagues found that patients with cancer presented an important risk of secondary events due to COVID-19 if we compare it with a population without cancer: $39 \%$ in the oncologic population versus $8 \%$, hazard ratio of 3.56 . Zhang and colleagues in a retrospective analysis identified 28 patients positive for COVID and with a cancer among 1,276 patients admitted in hospital. This prevalence $(2.2 \%)$ is 1.7 (95\% confidence interval, 1.2, 2.4) times higher than the Chinese population of the same age (4). The probability of dying for COVID with a cancer was of $28.6 \%$ (5). Zhang found that the administration of anticancer therapies was an independent predictor of death. They also described the high proportion of patients who acquired the infection in hospital, when they came for cancer treatment.

Although these data included a very small and heterogeneous sample of patients with cancer, reports from Italy confirm the potential higher risk of COVID-19 infection in patients with cancer, indicating that the $20 \%$ of patients dead for COVID-19 infection also had a concomitant diagnosis of cancer (6). More recently, clinical data on COVID-19 cases from two hospitals in New York City (7) observed that 23 out of the 393 (5.9\%) reported cases were patients with cancer, and 10 of them required invasive mechanical ventilation (representing $7.7 \%$ of the total of patients requiring 
invasive mechanical ventilation). In another institution from New York City, 28\% of COVID-19 + cancer patients died from COVID-19 with a case fatality rate of $37 \%$ for hematologic malignancies and $25 \%$ for solid malignancies. This study reported that in this population, older age, multiple comorbidities, need for ICU support, and elevated levels of Ddimers, LDH, and lactate predicted poorer outcome (7).

Therefore, healthcare professionals were rapidly faced with the challenge of profoundly re-organizing healthcare systems at unprecedented pace during the COVID-19 crisis to balance the competing risks of death from cancer $v s$. death or serious complications from infection.

The Chinese series suggested that postponing adjuvant chemotherapy or elective surgery for less aggressive cancers should be considered. Furthermore, more intensive care should be done for the patients with cancer who are infected (3). These measures have been later taken by healthcare organizations like ASCO and ESMO who have deepened these recommendations extending it to postponing clinics or balancing the cost/benefit ratio according to outcome, with prioritization of adjuvant therapies (8). Similarly, the benefits and risks of palliative therapies and the options of temporary stopping the therapy and switch to oral drugs, if available, during the pandemic needed to be considered. Recommendations for management of cancer patients in clinical trials have also been made available by different regulatory agencies (9). However, as this outbreak prolongs in time and with the unknown risk-benefit balance between undertreating patients with cancer (with resultant increase in cancer-related morbidity and mortality) and preventing the expected higher morbidity and mortality from COVID-19, initiation of systemic cancer therapy seems unavoidable.

The scarce of systematic information on prevalence and incidence in overall population toughen the possibility of real comparisons with patients with cancer although contagiousness rates seem rather high. For example, estimates on infection rates vary from $0.76 \%$ for residents of Iceland to $36 \%$ for residents of Boston. This likely overstates the overall population mean, which some observers have suggested is around 40\% (10).

Systematic reports about the prevalence of cancer in patients with COVID-19 and the real incidence of COVID-19 infection among cancer patients are starting to be made public. A review and meta-analysis reported by Desai et al. (11) found 11 studies of patients with COVID-19 with the data of cancer prevalence: $2 \%$ (95\% CI 2.0 to $3.0 \%: \mathrm{I}^{2}=83.2 \%$ ) in patients treated for COVID-19. A similar meta-analysis by Emami et al. (12) reported a prevalence of malignancy of $0.92 \%$ (95\% CI, $0.56-$ $1.34 \%)$. In a recent dedicated session to cancer patients and COVID-19 infection during the last AACR annual symposium, several cancer institutions and hospitals across the world presented updates on their management and outcome of cancer patients with COVID-19 infection. These series reported variability within different countries in terms of incidence and prevalence. For example, in our own series, of Gustave Roussy Hospital, including more than 1,300 tested cancer patients, COVID-19 positivity was observed in around $12 \%$, whereas retrospective series including 1,524 cancer patients from Wuhan reported a COVID-19 positivity rate of only $0.79 \%$ (13).

The standard method to diagnose infection by COVID-19 is through identification by RT-PCR SARS-CoV-2 testing. Since SARS-CoV-2 is usually transmitted by the upper respiratory tract, mostly swab samples are taken from the nasopharynx. It has also been accepted to perform the swabs directly to the oropharynx, although some studies suggest that the nasopharynx would be somewhat more sensitive than the oropharynx (14). Positivity from nasopharynx swabs is variable and ranges from 53.6 to $73.3 \%$ depending on the series (15). There are several factors that might affect the performance (sensitivity and specificity) of the test like the quality of the sample, the sampling technique, transportation process, or limited gene detection. In fact, it has been shown that high viral loads soon after symptom onset, which then gradually decreased towards the detection limit at about day 21, with no obvious difference in viral loads across sex, age groups, and disease severity (16). In fact, similar viral loads have been documented in the upper respiratory tract of both symptomatic and asymptomatic cases (17) and in the pre-symptomatic phase (18).

Several strategies are available in order to increase sensitivity and specificity of this testing. In a report of 67 patients with confirmed COVID-19 infection, the duration of positive test in nasopharyngeal swabs has a median of 12 days (range, 3-38), in sputum of 19 days (range, 5-37), and in stools of 18 days (range, 7-26). SARS-CoV-2 RNA was detectable for a duration of 30 days (19). After a negative test of nasopharyngeal swabs among 46 patients, $28(60.9 \%)$ and $14(30.4 \%)$ patients were still positive in sputum and stools.

Another approach consists in the realization of chest CT scans in patients suspected or tested for COVID-19 in addition to RT-PCR for nasal or oropharynx swabs. Consistently between patients' series, the main symptoms associated to SARS-CoV-2 infection in addition to fever is the presence of pulmonary symptoms ranging from dry cough to pneumonia up to acute distress respiratory syndrome leading to death (3). Therefore initially it was suggested that lung cancer patients or patients who had suffered previous lung surgery would be at higher risk of lung complications from SARS-CoV-2 so more intensive followup and chest CT scrutiny should be required for these patients. Initial data from Wuhan series (20) indeed reported a higher risk of COVID-19 infection among their cancer patients (7 out of 28; $25 \%)$. A recent international series compilation of 200 patients with thoracic tumors affected with COVID-19 (21) reported an extremely high death rate of $34.6 \%$, mostly due to acute respiratory distress syndrome and multi-organ failure. These findings have not been replicated by other series like Wuhan reports (22) where mortality was not affected by type of cancer (any cancer $v s$. lung cancer; $\mathrm{HR}=0.727 ; \mathrm{p}=0.589$ ). What it has been noteworthy is that chest CT demonstrates typical radiographic features in almost all COVID-19 patients irrespective of presence of cancer or type of cancer. These include ground-glass opacities, multifocal patchy consolidation, and/or interstitial changes with a peripheral distribution (23). Those typical pulmonary abnormalities were also observed in 
patients with negative RT-PCR results but clinical symptoms in small-scale studies (24).

A report of the correlation of chest CT and RT-PCR testing in 1,014 cases from China found a RT-PCR positivity in 59\% ( $\mathrm{n}=$ $601)$ of the patients and a chest CT positivity of $88 \%(n=888)$. The negative RT-PCR results is correlated to a $75 \%(\mathrm{n}=308)$ with positive chest CT findings. The combination of RT-PCR and chest CT gave respectively a sensitivity, specificity, and accuracy of $97 \%(n=580), 25 \%(n=105)$, and 68\% ( $n=685)$, a positive predictive value of $65 \%(n=580)$, and a negative predictive value of $83 \%(n=105)$ (25). Another work analyzed 1,099 hospitalized patients with a positive test for COVID-19: $86 \%(n=840)$ of patients had CT imaging with finding of ground-glass opacity, local patchy shadowing, or interstitial pneumopathies; $17.9 \%(\mathrm{n}=157)$ of patients has no radiographic abnormality (26).

Despite the standardized use of CT scans in addition to (or instead of) RT-PCR for the diagnoses of a suspected COVID-19 infection might still be controversial (27). First, the findings on chest imaging in COVID-19 are indeed not specific, and overlap with other infections, including influenza, H1N1, SARS, and MERS. Second, there are issues related to cleaning imaging equipment to control the spread of infection in health care facilities where CT scans are frequently used. For instance, portable radiography units are less expensive, can be cleaned easier, and could be an alternative. Chest CT scan might provide prognostic information as, some published data reported that the presence of a patchy consolidation by lung CT scan at patient admission was associated to possibility of a severe event in a multivariate analysis in COVID-19+ patients $(\mathrm{HR}=5.438$; CI 1.498-19.748; $\mathrm{p}=0.010$ ). In addition, it might prove useful for the management of patients with COVID-19 infection, especially in highly symptomatic cases. Indeed, as additional knowledge of this infection becomes available, several reports have shown that COVID-19 infection might be associated to an inflammatory syndrome evidenced by high levels of inflammatory markers and increased risk of thromboembolism associated to this infection (28). This might be important in patients with cancer with already increased phenomena of coagulopathy and thrombosis. Hence, the interest of associating chest imaging in cancer patients to identify underlying pulmonary embolism, which might contribute to worsen respiratory symptoms and require specific additional treatment (29). Beyond the role of CT scan without intravenous contrast agent injection for the diagnostic workup, prognostic evaluation, and follow-up of COVID-19 infection, selected patients may benefit from contrast enhanced CT pulmonary angiography to diagnose potentially lifethreatening pulmonary embolism and start appropriate therapies. A study reported a high frequency of either pulmonary embolism in critically ill ICU patients with COVID-19 complications [7.1, 20.6 (30), and 49\% (31)].

Rogado et al. detected 45/1,069 COVID-19 diagnoses in cancer patients $v s 42,450 / 6,662,000$ in total population $(\mathrm{p}<0.00001)$ in a Spanish hospital. Mortality rate: 19/45 cancer patients $v s 5,586 / 42,450(\mathrm{p}=0.0001)$. Mortality was associated with older median age, adjusted by staging, and histology (74 vs 63.5 years old, OR 1.06, $\mathrm{p}=0.03$ ). Patients who combined hydroxychloroquine and azithromycin presented 3/18 deaths, regardless of age, staging, histology, cancer treatment, and comorbidities (OR 0.02, p = 0.03) (32).

In a Spanish series Mestre-Gomez et al. retrospectively reviewed 452 electronic medical records of patients admitted to Internal Medicine Department of a secondary hospital in Madrid during COVID-19 pandemic outbreak. Ninety-one patients had a Computed Tomography pulmonary angiography (CTPA). The cumulative incidence of $\mathrm{PE}$ was assessed with a clinical, analytical, and radiological characterization compared in patients with and without PE. The incidence of PE was $6.4 \%$. They evaluated the D-dimer peak and they found a significant elevation in PE vs non-PE patients $(14,480$ vs $7,230 \mathrm{mcg} / \mathrm{dl}$, $\mathrm{p}=0.03$ ). In multivariate analysis that plasma $\mathrm{D}$-dimer peak was confirmed as an independent predictor of PE with a best cut off point of $>5,000 \mu \mathrm{g} / \mathrm{dl}(33)$.

In a hematologic series at the University Hospital Freiburg, Shoumariyeh et al. analyzed a retrospective cohort of 39 patients with hematological and solid cancers hospitalized for COVID19. With univariate and multivariate Cox analysis they found that the presence of a malignancy was not significantly associated with survival or severe events. Instead the high IL- 6 levels at COVID-19 diagnosis $(\mathrm{HR}=6.95, \mathrm{P}=.0121)$ and age $\geq 65$ years ( $\mathrm{HR}=6.22, \mathrm{P}=.0156)$ were related to mortality. Another find of Shoumariyeh et al. was about patients with a hematological malignancy that showed a longer duration of clinical improvement and longer hospitalization compared to patients with a solid cancer (34).

In our institution, we have therefore taken the decision to perform RT-PCR SARS-CoV-2 testing by nasopharyngeal swab in a specifically dedicated area to our patients at day -2 or -1 before administration of chemotherapy or immunotherapy treatment, if feasible. The reason is to discover the disease in its presymptomatic phase to prevent initiation of a potentially immunosuppressive treatment to diminish chances of major complications and to be able to intensively follow up these patients. In addition, identifying those pre-symptomatic and asymptomatic patients will help rapidly isolate these COVID-19+ patients to prevent further spreading of the disease to health personnel and to other patients. For those patients with respiratory symptoms a chest CT scan is also performed to increase sensitivity to the diagnoses of the disease. In addition, CT pulmonary angiogram is performed for those cases with acute inflammatory syndrome to rule out pulmonary embolism and to initiate intensive anticoagulation therapy. For patients confirmed COVID-19+, systemic treatments are delayed and patient is surveyed or treated accordingly. All COVID-19+ cases will be retested at around 15 days from the initial testing. For those patients asymptomatic throughout the infection with a negative test at 15 days, systemic treatment can be initiated. In case of a symptomatic course of the infection, systemic anticancer treatment can be started around 15 days after the end of symptoms. If the second PCR test still is positive, patients need to be rested at 7-15 days and systemic treatment must be delayed if possible. For those cases still COVID-19+ after at least 15 days from the diagnostic PCR, 
chemotherapy can be initiated if the patient has no symptoms for at least 7 days for those cases where benefit/risk is in favor for cancer therapy. Soon, as validated RT-PCR SARS-CoV-2 testing becomes available in local laboratories, testing will be performed at the same time as the standard pre-chemotherapy blood samples before every cycle of treatment, with results being directly reported to our institution.

In the near future, the availability of laboratory IgM or IgG validated testing to evaluate the previous SARS-CoV-2 exposure will be helpful in order to fully picture the real incidence of this disease in overall population and more particularly in cancer patients. This will become crucial if further studies observe the

\section{REFERENCES}

1. Kamboj M, Sepkowitz KA. Nosocomial Infections in Patients With Cancer. Lancet Oncol (2009) 10:589-59. doi: 10.1016/S1470-2045(09)70069-5

2. Sica A, Massarotti M. Myeloid Suppressor Cells in Cancer and Autoimmunity. J?Autoimmun (2017) 85:117-25. doi: 10.1016/ j.jaut.2017.07.010

3. Liang W, Guan W, Chen R, Wang W, Li J, Xu K, et al. Cancer Patients in SARS-CoV-2 Infection: A Nationwide Analysis in China. Lancet Oncol (2020) 21:335-7. doi: 10.1016/S1470-2045(20)30096-6

4. Ferlay J, Ervik M, Lam F. Global Cancer Observatory: Cancer Today. Int Agency Res Cancer (2018) 2:435-54. doi: 10.1002/ijc.33232

5. Wu Z, McGoogan JM. Characteristics of and Important Lessons From the Coronavirus Disease 2019 (COVID-19) Outbreak in CHINA: Summary of a rEport of 72314 Cases From the Chinese Center for Disease Control and Prevention. JAMA (2020) 2:321-42. doi: 10.1001/jama.2020.2648

6. Onder G, Rezza G, Brusaferro S. Case-Fatality Rate and Characteristics of Patients Dying in Relation to COVID-19 in Italy. JAMA (2020) 3:654-6. doi: 10.1001/jama.2020.4683

7. Mehta V, Goel S, Kabarriti R, Cole D, Goldfinger M, Acuna-Villaorduna A, et al. Case Fatality Rate of Cancer Patients with COVID-19 in a New York Hospital System. Cancer Discov (2020) 10:935-41. doi: 10.1158/21598290.CD-20-0516

8. Hanna TP, Gerald GA, Christopher CM. Cancer, COVID-19 and the Precautionary Principle: Prioritizing Treatment During a Global Pandemic. Nat Rev (2020) 17:268-70. doi: 10.1038/s41571-0200362-6

9. Bruno de Paula H, Araújo I, Bandeira L, Barreto NMPB, Doherty GJ. Recommendations From National Regulatory Agencies for Ongoing Cancer Trials During the COVID-19 Pandemic. Lancet Oncol (2020) 8):455-7. doi: 10.1016/S1470-2045(20)30226-6

10. Oran P, Topol J. Getting a Handle on Asymptomatic SARS-CoV-2 Infection. Scripps Res (2020) 5:678-9. doi: 10.7326/M20-3012

11. Desai R. COVID-19 and Cancer: Lessons from a Pooled Meta-Analysis. JCO (2020) 8:557-9. doi: 10.1200/GO.20.00097

12. Song Y, Xia J, Guo Q, Song T, He J, Peiris M. Prevalence of Underlying Diseases in Hospitalized Patients with COVID-19: A Systematic Review and Meta-Analysis. Arch Acad Emerg Med (2020) 8:765-70. doi: 10.1016/ j.ajem.2020.10.022

13. Yu J, Ouyang W, Chua MLK, Xie C. SARS-CoV-2 Transmission in Patients with Cancer at a Tertiary Care Hospital in Wuhan, China. JAMA Oncol (2020) 25:34-8. doi: 10.1001/jamaoncol.2020.0980

14. Zou L, Ruan F, Huang M, Liang L, Kang M. SARS-CoV-2 Viral Load in Upper Respiratory Specimens of Infected Patients. N Engl J Med (2020) 382:1177-9. doi: 10.1056/NEJMc2001737

15. Yang Y, Yang M, Shen C. Evaluating the Accuracy of Different Respiratory Specimens in the Laboratory Diagnosis and Monitoring the Viral Shedding of 2019-nCoV Infections. Nat (2020) 2:212-5. doi: 10.1101/2020.02. 11.20021493

16. He X, Lau E, Wu P. Temporal Dynamics in Viral Shedding and Transmissibility of COVID-19. Nat Med (2020) 4:665-7. doi: 10.1038/ s41591-020-0869-5 acquisition of an adaptive immunity capable of preventing reinfection to SARS-CoV-2 infection, or at least to severe forms to allow treating cancer patients without additional risk from COVID-19.

\section{AUTHOR CONTRIBUTIONS}

$\mathrm{AV}$ and MA: oncologist point of view and writing and bibliographic search. SA and LD: radiologist point of view and writing and figure creation. All authors contributed to the article and approved the submitted version.

17. Hoehl S, Rabenau H, Berger A. Evidence of SARS-CoV-2 Infection in Returning Travelers From Wuhan, China. N Engl J Med (2020) 4:1278-80. doi: 10.1056/NEJMc2001899

18. Bhangu A, Lawani I, Ng-Kamstra JS, Wang Y, Chan A. Global Guidance for Surgical Care During the COVID-19 Pandemic. Br J Surg (2020) 15:898-1. doi: $10.1002 /$ bjs. 11646

19. Horn L, Whisenant JG, Torri V, Huang L-C, Trama A, Paz-Ares LG, et al. Thoracic Cancers International COVID-19 Collaboration (TERAVOLT): Impact of Type of Cancer Therapy and COVID Therapy on Survival. J Clin Oncol (2020) 38:(18 Suppl). doi: 10.1200/JCO.2020.38.18_suppl.LBA111

20. Zhang L, Zhu F, Xie L, Wang C, Wang J, Chen R, et al. Clinical Characteristics of COVID-19-Infected Cancer Patients: A Retrospective Case Study in Three Hospitals Within Wuhan, China. Ann Oncol (2020) 3:363-70. doi: 10.1016/ j.annonc.2020.03.296

21. Mitchell RL, Blakely LJ, Schleicher SM, Poole SL, Dickson NR, Patton J, et al. Maintaining Treatment Volumes During the COVID-19 Pandemic. J Clin Oncol (2020) 38(suppl 29; abstr 103). doi: 10.1200/JCO.2020.38.29_suppl.103

22. Xie X, Zhong Z, Zhao W. Chest CT for Typical 2019-nCoV Pneumonia: Relationship to Negative RT-PCR Testing. Radiology (2020) 6:65-74. doi: 10.1148/radiol.2020200343

23. Chung M, Bernheim A, Mei X. CT Imaging Features of 2019 Novel Coronavirus (2019-nCoV). Radiology (2020) 7:660-7. doi: 10.1148/ radiol.2020200230

24. Huang P, Liu T, Huan L. Use of Chest CT in Combination with Negative RTPCR Assay for the 2019 Novel Coronavirus but High Clinical Suspicion. Radiology (2020) 8:445-553. doi: 10.1148/radiol.2020200330

25. Ai T, Yang Z, Hou H, Zhan C, Chen C, Lv W, et al. Correlation of Chest CT and RT-PCR Testing for Coronavirus Disease 2019 (COVID-19) in China: A Report of 1014 Cases. Radiology (2020) 8:32-40. doi: 10.1148/ radiol.2020200642

26. Guan W, Zheng N, Wen L. Clinical Characteristics of Coronavirus Disease 2019 in China. NEJM (2020) 4:67-74. doi: 10.1056/NEJMoa2002032

27. Hope A. A role for CT in COVID-19? What Data Really Tell us so Far. Lancet (2020) 395:665-78. doi: 10.1016/S0140-6736(20)30728-5

28. Klok FA, Kruip M, vanderMeer N. Incidence of Thrombotic Complications in Critically ill ICU Patients with COVID-19. Thromb Res (2020) 4:323-45. doi: 10.1016/j.thromres.2020.04.013

29. Zhang Y, Xiao M, Zhang S, Xia P. Coagulopathy and Antiphospholipid Antibodies in Patients with COVID-19. N Engl J Med (2020) 8:567-34. doi: 10.1056/NEJMc2007575

30. Poissy J, Goutay J, Caplan M, Parmentier E, Duburcq T, Lassalle F, et al. Pulmonary Embolism in COVID-19 Patients: Awareness of an Increased Prevalence. CIRCULATION (2020) 142:184-6. doi: 10.1161/ CIRCULATIONAHA.120.047430

31. Klok FA, Kruipb MJHA, van der Meercd NJM, Arbouse MS, Gommersf D, Kantg KM, et al. Confirmation of the High Cumulative Incidence of Thrombotic Complications in Critically ill ICU Patients with COVID-19: An Updated Analysis. Thromb Res. (2020) 191:148-50. doi: 10.1016/ j.thromres.2020.04.041

32. Rogado J, Obispo B, Pangua C, Serrano-Montero G, Martín Marino A, PérezPérez M, et al. COVID-19 Transmission, Outcome and Associated Risk Factors in Cancer Patients at the First Month of the Pandemic in a Spanish 
Hospital in Madrid. Clin Trans Oncol (2020) 22:2364-8. doi: 10.1007/s12094020-02381-z

33. Mestre-Gómez B, Lorente-Ramos RM, Rogado J, Franco-Moreno A, Obispo B, Salazar-Chiriboga D, et al. Incidence of Pulmonary Embolism in Non-Critically Ill COVID-19 Patients. Predicting Factors for a Challenging Diagnosis. J Thromb Thrombolysis (2020) 1-7. doi: 10.1007/s11239-020-02190-9

34. Shoumariyeh K, Biavasco F, Ihorst G, Rieg S, Nieters A, Kern WV, et al. COVID-19 in Patients with Hematological and Solid Cancers at a Comprehensive Cancer Center in Germany. Cancer Med (2020) 22):841222. doi: $10.1002 / \mathrm{cam} 4.3460$
Conflict of Interest: The authors declare that the research was conducted in the absence of any commercial or financial relationships that could be construed as a potential conflict of interest.

Copyright (๑) 2021 Viansone, Ammari, Dercle and Arnedos. This is an open-access article distributed under the terms of the Creative Commons Attribution License (CC BY). The use, distribution or reproduction in other forums is permitted, provided the original author(s) and the copyright owner(s) are credited and that the original publication in this journal is cited, in accordance with accepted academic practice. No use, distribution or reproduction is permitted which does not comply with these terms. 